\title{
Assessing President Obama's Appointment of Women to the Federal Appellate Courts
}

\author{
Laura P. Moyer*
}

\begin{abstract}
A major legacy of the Obama presidency was the mark he left on the federal courts with respect to increasing judicial diversity. In particular, President Obama's appointments of women to the federal judiciary exceeded all previous presidents in terms of both absolute numbers and as a share of all judges; he also appointed a record-setting number of women of color to the lower federal courts. In this Article, I take an intersectional approach to exploring variation in the professional backgrounds, qualifications, and Senate confirmation experiences of Obama's female appeals court appointees, comparing them with George W. Bush and Bill Clinton appointees. These data reveal that women of color appointed by Obama differ from both white women and minority men in terms of ABA ratings, the types of professional experiences they bring with them, and whether they were confirmed by a roll call vote.
\end{abstract}

\section{KEYWORDS}

Federal Judges, Intersectionality, Diversity, Obama, Women

\section{CONTENTS}

Assessing President Obama's Appointment of Women to the Federal Appellate Courts.

I. Appointing Women Across Three Presidents: Professional

EXPERIENCES AND QUALIFICATIONS ...................................................438

A. Obama's Female Nominees ......................................................438

B. Legal Education and ABA Ratings .........................................439

C. Law Clerk Experience ......................................................... 441

D. Judicial and Prosecutorial Experience....................................442

E. Private practice ............................................................ 445

F. Academia ............................................................................ 445

* University of Louisville, laura.moyer@louisville.edu.

Data for this project were collected with support from the National Science Foundation (NSF SES \#1655159). Any opinions, findings, and conclusions or recommendations expressed are those of the author and do not necessarily reflect the views of the National Science Foundation. 
II. Running the Gauntlet: The Senate Confirmation Process .........446

A. Changes in the Senate Confirmation Process ...............................446

B. Assessing the Treatment of Obama's Female Nominees in the Senate 450

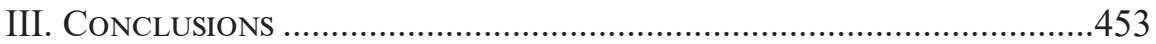




\section{Assessing President Obama’s Appointment of Women to The Federal ApPellate Courts}

The underrepresentation of women on the federal bench has been a source of concern for presidents of both parties over the past forty years, ${ }^{1}$ but no president has done more to advance the cause of gender equity than Barack Obama. Like Clinton ${ }^{2}$ and Carter, ${ }^{3}$ Obama made a public commitment to improving the representation of women and minorities on the federal bench ${ }^{4}$ and was successful in following through with that commitment. ${ }^{5}$ Obama's appointments of women to the lower federal courts exceeded all previous presidents in terms of both absolute numbers and as a share of all judges; he also appointed two women to the U.S. Supreme Court, more than any other president. Additionally, Obama appointed a recordsetting number of women of color, including the first Asian American woman to a federal appeals court and the first Native American woman to the federal district courts. At the start of Obama's second term in office, the liberal interest group Alliance for Justice noted that six states had now gained their first female circuit court judge, and that two circuits (the First and the Sixth) had gained their first African-American woman judge. ${ }^{6}$ To illustrate these historic accomplishments in context, Figure 1 compares Obama's appointments to the U.S. Courts of Appeals with those of his two predecessors, George W. Bush and Bill Clinton.

The context in which these nominations occurred was one of persistent gender inequality in the legal profession. A report by the American Bar Association that was issued the year that Obama took office detailed a series of concerning statistics related to women. ${ }^{7}$ While women had very nearly achieved parity with men in attending and graduating from law school, there were significant gender gaps evident in wages and in a variety of employment settings. In private practice, women made up about 45 percent of associates but only 19 percent of partners. Women were vastly underrepresented in the ranks of general counsel of Fortune 500 companies

1 Susan B. Haire \& Laura P. Moyer, Diversity Matters: Judicial Policy Making in the U.S. Courts of Appeals, 2-5 (2015).

2 See, e.g., Sheldon Goldman \& Matthew D. Saronson, Clinton's Nontraditional Judges: Creating a More Representative Bench, 78 Judicature 68 (1994).

3 Sheldon Goldman, Picking Federal Judges: Lower Court Selection from Roosevelt through Reagan 236-284 (1997); Elliot E. Slotnick, Lowering the Bench or Raising It Higher: Affirmative Action and Judicial Selection during the Carter Administration, 1 Yale L. \& Pol'y Rev. 270, 275-282 (1983).

4 T.J. Kimel \& Kirk A. Randazzo, Shaping the Federal Courts: The Obama Nominees, 93 Soc. Sci. Q. 1243, 1249 (2012).

5 Elliot E. Slotnick et al., Obama's Judicial Legacy: The Final Chapter, 5 J. L. \& Cts. 363, 389-410 (2017); Rorie Spill Solberg \& Jennifer Segal Diascro, A Retrospective on Obama's Judges: Diversity Intersectionality, and Symbolic Representation, 2018 Pol. Groups \& Identities 1, 5. President Reagan also made a campaign pledge to nominate a woman to the Supreme Court, which he fulfilled with the successful confirmation of Sandra Day O'Connor. Haire \& Moyer, supra note 1, at 4.

6 Making History: President Obama's Female Judicial Nominees, Alliance for Justice (June 11, 2013), https://web.archive.org/web/20190331181355/https://www.afj.org/wpcontent/uploads/2013/06/President-Obama-Female-Firsts.pdf.

7 Am. Bar Ass'n: Comm'n on Women in the Profession, A Current Glance at Women in the Law 2009, 1-4 (2009), https://www.americanbar.org/content/dam/aba/administrative/ women/CurrentGlanceStatistics2009.pdf [https://perma.cc/XPW2-JV8G]. 


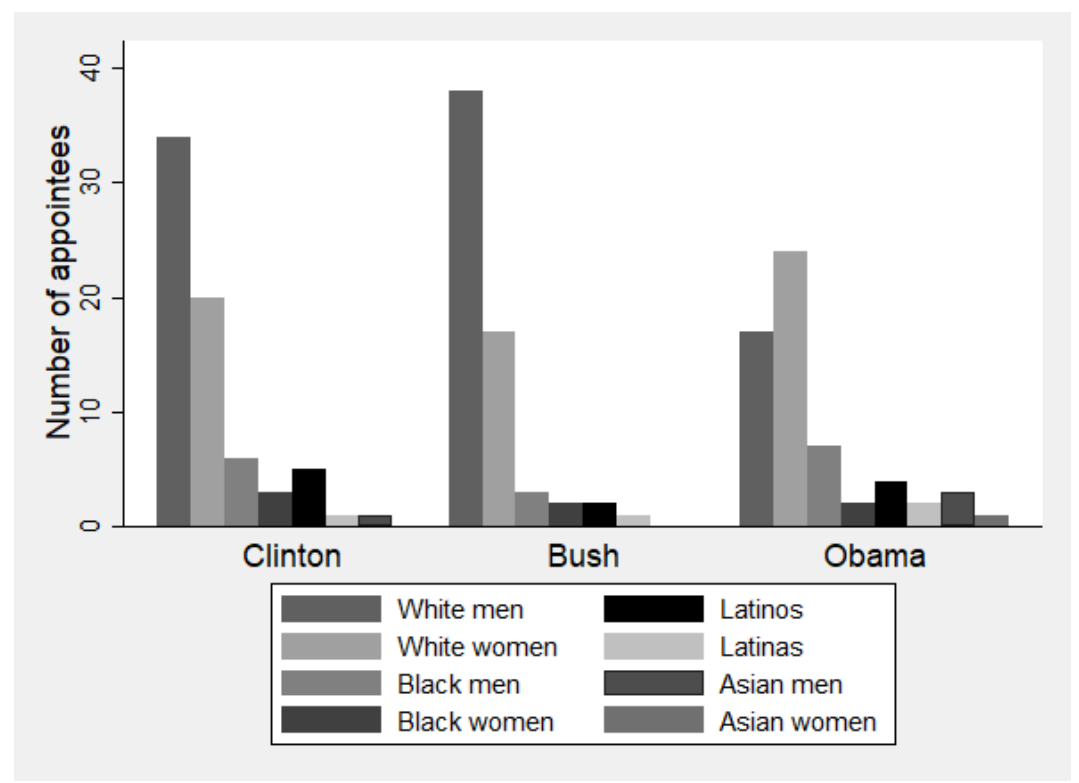

Figure 1: Race and Gender of Circuit Court Appointees.

By president (1992-2016)

(comprising 15\%) and as deans of law schools (20\%). ${ }^{8}$ Other studies documented persistent obstacles faced by women of color in the legal profession, creating disadvantages not felt by white women and that hampered their advancement in a variety of legal settings. ${ }^{9}$

The approach that the Obama White House took to diversifying the bench was two pronged, emphasizing both demographic and experiential diversity. Kathryn Ruemmler, who served as White House Counsel from 2011 to 2014, described Obama's philosophy this way: "Diversity in and of itself is a thing that is strengthening the judicial system. It enhances the bench and the performance of the bench and the quality of discussion ... to have different perspectives, different life experiences, different professional experiences, coming from a different station in life." ${ }^{10}$ Elsewhere, Ruemmler added that "the president wants the federal courts

8 By Obama's last year office, the American Bar Association report showed only limited progress on most of these indicators. A few areas showed substantial movement toward parity; women now made up nearly a quarter of lawyers in Fortune 500 general counsel jobs, and 31 percent of law school deans.

9 Carla D. Pratt, Sisters in Law: Black Women Lawyers'Struggle for Advancement, 2012 Mich. St. L. Rev. 1777, 1782-1790 (2014); Janet E. Gans Epner, Am. Bar Ass'n: Comm'n on Women in the Profession, Visible Invisibility: Women of Color in Law Firms, 9-37 (2006), http://www.nextions.com/wp-content/uploads/2017/05/visible-invisibility-abacommission-on-women-in-the-profession.pdf [https://perma.cc/8CAK-KLJB].

10 Philip Rucker, Obama Pushing to Diversify Federal Judiciary Amid GOP Delays, Wash. Post (Mar. 3, 2013), https://www.washingtonpost.com/politics/obama-pushingto-diversify-federal-judiciary-amid-gop-delays/2013/03/03/16f7d206-7aab-11e2-9a75dab0201670da_story.html [https://perma.cc/A3WU-V9GP]. This was consistent with 
to look like America. He wants people who are coming to court to feel like it's their court as well." 11 In a 2014 interview, Obama characterized the makeup of the federal bench under his tenure as something that "speaks to the larger shifts in our society, where what's always been this great American strength - this stew that we are- is part and parcel of every institution, both in the public sector as well as in the private sector." 12

Within the federal judiciary, the U.S. Courts of Appeals have increasingly become a focal point for presidents looking to cement their legacies. Judges who sit on these intermediate appellate courts frequently rule on legal issues of first impression and can influence how major legislative achievements of a president are actually implemented. For Obama, his appointments to these courts would play a major role in cases involving the Affordable Care Act, LGBT rights, voting rights, and other issues. While nominations to the U.S. Supreme Court receive the lion's share of attention from the general public, interest groups and both political parties have increasingly turned their attention to the individuals that presidents nominate to life tenured seats on these federal appeals courts. ${ }^{13}$ It is also worth noting that, as of the date of this writing, eight of the nine justices currently sitting on the Supreme Court previously served as judges in the U.S. Courts of Appeals. Thus, the stakes are high when a president has an opportunity to make a nomination.

In this Article, I describe the professional backgrounds and confirmation experiences of the women nominated by President Obama and confirmed by the Senate to the federal appellate bench. Drawing on data from the Federal Judicial Center and Holmes, Shomade, and Hartley, ${ }^{14}$ I utilize an intersectional approach ${ }^{15}$ to assess how white women and women of color's experiences compare with each other, as well as with the men in their appointment cohort. To evaluate the extent to which the Obama appointments were distinctive, I also compare Obama's female judges with women appointed by George W. Bush and Bill Clinton. Then, in the final part of the chapter, I discuss how the political environment of the Obama era, characterized by extreme polarization and contentiousness in confirmations, affected the confirmation experiences of women nominated to the U.S. Courts of Appeals, relative to women nominated by previous presidents.

the 2008 platform of the Democratic Party, which emphasized the selection of judges "who represent the diversity of America." Haire \& Moyer, supra note 1, at 131.

11 John Schwartz, For Obama, a Record on Diversity but Delays on Judicial Confirmations, N.Y. Times (Aug. 6, 2011), https://www.nytimes.com/2011/08/07/us/politics/07courts. html [https://perma.cc/8F76-V8TN].

12 Jeffrey Toobin, The Obama Brief: The President Considers his Judicial Legacy, New Yorker (Oct. 20, 2014), https://www.newyorker.com/magazine/2014/10/27/obama-brief [https://perma.cc/9VUE-5LYE].

13 Nancy Scherer et al., Sounding the Fire Alarm: The Role of Interest Groups in the Lower Federal Court Confirmation Process, 70 J. Pol. 1026, 1027 (2008).

14 See generally Lisa M. Holmes et al., The Confirmation Obstacle Course: Signaling Opposition Through Delay, 33 Am. Rev. Pol. 23 (2012).

15 See generally Ange-Marie Hancock, Intersectionality: An Intellectual History (2016). The project was made possible in part through the support of the National Science Foundation (NSF-SES \#1655159). I also would like to acknowledge Lisa Holmes for generously sharing her data on Clinton and Bush nominees' committee delay. 
Table 1: Female appeals court judges appointed by Obama (2009-2016).

\begin{tabular}{|c|c|c|}
\hline Judicial Appointee & Circuit & Date Nominated \\
\hline Julie E. Carnes & 11 & January 6,2014 \\
\hline Susan Laura Carney & 2 & January 5, 2011 \\
\hline Morgan Christen & 9 & May 18, 2011 \\
\hline Bernice Bouie Donald & 6 & January 5, 2011 \\
\hline Michelle Taryn Friedland & 9 & January 6,2014 \\
\hline Pamela Ann Harris & 4 & May 8, 2014 \\
\hline Barbara Milano Keenan & 4 & September 14, 2009 \\
\hline Jane Louise Kelly & 8 & January 31,2013 \\
\hline Cheryl Ann Krause & 3 & February 6, 2014 \\
\hline Beverly Baldwin Martin & 11 & June 19, 2009 \\
\hline Carolyn Baldwin McHugh & 10 & January 6,2014 \\
\hline Patricia Ann Millett & D.C. & June 4, 2013 \\
\hline Nancy Louise Moritz & 10 & January 6,2014 \\
\hline Mary Helen Murguia & 9 & March 25, 2010 \\
\hline Jacqueline Hong-Ngoc Nguyen & 9 & September 22, 2011 \\
\hline Kathleen McDonald O’Malley & Fed. & March 10, 2010 \\
\hline Cornelia Thayer Livingston Pillard & D.C. & June 4, 2013 \\
\hline Jill Anne Pryor & 11 & January 6,2014 \\
\hline Robin Stacie Rosenbaum & 11 & January 6,2014 \\
\hline Patty Shwartz & 3 & January 4, 2013 \\
\hline Kara Farnandez Stoll & Fed. & January 7,2015 \\
\hline Jane Branstetter Stranch & 6 & August 6, 2009 \\
\hline Stephanie Dawn Thacker & 4 & September 8, 2011 \\
\hline Ojetta Rogeriee Thompson & 1 & October 6, 2009 \\
\hline
\end{tabular}

\section{Appointing Women Across Three Presidents: PROFESSIONAL EXPERIENCES AND QUALIFICATIONS}

\section{A. OBAMA'S FEMALE NOMINEES}

Table 1 lists each woman nominated by Obama who was confirmed to a life-tenured federal appellate judgeship, the date of the nomination, and the circuit in which they were seated. Although the Obama administration was the subject of some criticism for a slow start in nominations to the lower federal courts, ${ }^{16}$ in his first year in office, Obama nominated four women, including one African American woman (Ojetta

16 Sheldon Goldman et al., Obama's Judiciary at Midterm: The Confirmation Drama Continues, 94 Judicature 262, 263-264 (2011). 
Rogeriee Thompson) to the First Circuit. ${ }^{17}$ This was followed by two women in 2010, five in 2011, four in 2013, eight in 2013, and one in 2015.

Over the course of his two terms, Obama influenced some circuits more substantially than others when it came to improving gender diversity. ${ }^{18}$ The Ninth Circuit added four women, as did the Eleventh Circuit; Obama's appointments were more consequential for the latter court, which has only 12 seats compared to the Ninth's 29 seats. Another court, the Fourth Circuit gained three women and, over the course of Obama's years in office, moved from a majority white-male court to one in which most of the judges were women, racial minorities, or both. ${ }^{19}$

On the U.S. Supreme Court, observers have noted the traditional of designating particular seats for judges of certain groups. For instance, there have been lengthy periods in which there was unofficially a "Jewish seat" and a "Catholic seat" on the Supreme Court. ${ }^{20}$ Thus, it seems reasonable that Obama might have approached the task of filling vacancies with an eye toward the identity of the previous occupant. In their analysis of presidential replacement strategies for the lower federal courts, Solberg and Diascro concluded that Obama did not tend to view particular seats as "belonging" to nontraditional judges, but rather adopted a broader strategy of diversification. $^{21}$

To further explore variation in the women that Obama appointed to the appeals court bench, the next section examines two indicators of qualifications that are a focus of the confirmation process: law school pedigree and American Bar Association ratings.

\section{B. LEGAL EDUCATION AND ABA RATINGS}

McGuire notes that the prestige of a law school education can affect the trajectory of an attorney's professional career, including membership in the Supreme Court bar. ${ }^{22}$ In particular, an Ivy League pedigree is likely to serve as a heuristic that signals a nominee is "high quality." Looking at Obama's successful appellate nominees, one third received their law degree from an Ivy League institution, similar to judges nominated by Bush and Clinton. ${ }^{23}$ Among Obama's 24 female nominees,

17 Thompson was the great-granddaughter of a white slave owner who had been indicted for his interracial marriage to a mixed-race enslaved woman. Judith Colenback Savage, Judicial Profile: Hon. Ojetta Rogeriee Thompson Judge, U.S. Court of Appeals for the First Circuit, Fed. Bar Ass'n (Sept. 2014), https://www.fedbar.org/wp-content/ uploads/2019/10/Thompson_Sept2014_5-pgs-pdf-3.pdf [https://perma.cc/HSP6NTG8].

18 With respect to other impacts on aspects of circuit composition, one analysis of Obama's first term identified seats in 7 of the 12 geographic circuits in which Obama's appointee replaced a judge appointed by a Republican judge. Susan B. Haire et al., Presidents and Courts of Appeal: The Voting Behavior of Obama's Appointees, 97 Judicature 137, 142 (2013).

19 Haire \& Moyer, supra note 1, at 132-134.

20 P. S. Ruckman, Jr., The Supreme Court, Critical Nominations, and the Senate Confirmation Process, 55 J. Pol. 793, 796 (1993).

21 Solberg \& Diascro, supra note 5, at 10.

22 Kevin McGuire, Lawyers and the U.S. Supreme Court: The Washington Community and Legal Elites, 37 Am. J. Pol. Sci. 365, 373-374 (1993).

23 Slotnick et al., supra note 5, at 400. Slotnick found that a higher proportion of Carter's 
Table 2: ABA Ratings.

\begin{tabular}{|c|c|c|c|c|c|c|c|c|}
\hline & \multicolumn{2}{|c|}{ All Judges } & \multicolumn{2}{|c|}{ Women } & \multicolumn{2}{|c|}{ Racial Minority } & \multicolumn{2}{|c|}{ White } \\
\hline & $\begin{array}{c}\text { All } \\
\text { women }\end{array}$ & $\begin{array}{c}\text { All } \\
\text { men }\end{array}$ & $\begin{array}{c}\text { Nonwhite } \\
\text { women }\end{array}$ & $\begin{array}{c}\text { White } \\
\text { women }\end{array}$ & Women & Men & Women & Men \\
\hline Obama & $\begin{array}{r}70.8 \% \\
(17 / 24) \\
\end{array}$ & $\begin{array}{c}87 \% \\
(27 / 31) \\
\end{array}$ & $\begin{array}{c}40 \% * \\
(2 / 5)\end{array}$ & $\begin{array}{l}78.9 \% * \\
(15 / 19)\end{array}$ & $\begin{array}{c}40 \% * * \\
(2 / 5) \\
\end{array}$ & $\begin{array}{c}85.7 \% * * \\
(12 / 14) \\
\end{array}$ & $\begin{array}{c}78.9 \% \\
(15 / 19) \\
\end{array}$ & $\begin{array}{r}88.2 \% \\
(15 / 17) \\
\end{array}$ \\
\hline GWB & $\begin{array}{r}70.6 \% \\
(12 / 17) \\
\end{array}$ & $\begin{array}{c}69.8 \% \\
(30 / 43) \\
\end{array}$ & $\begin{array}{c}66.7 \% \\
(2 / 3) \\
\end{array}$ & $\begin{array}{r}71.4 \% \\
(10 / 14) \\
\end{array}$ & $\begin{array}{c}66.7 \% \\
(2 / 3) \\
\end{array}$ & $\begin{array}{l}40 \% \\
(2 / 5) \\
\end{array}$ & $\begin{array}{c}71.4 \% \\
(10 / 14)\end{array}$ & $\begin{array}{r}73.7 \% \\
(28 / 38) \\
\end{array}$ \\
\hline Clinton & $\begin{array}{c}80 \% \\
(16 / 20)\end{array}$ & $\begin{array}{l}71.7 \% \\
(33 / 46)\end{array}$ & $\begin{array}{l}60 \% \\
(3 / 5)\end{array}$ & $\begin{array}{c}86.7 \% \\
(13 / 15)\end{array}$ & $\begin{array}{l}60 \% \\
(3 / 5)\end{array}$ & $\begin{array}{l}58.3 \% \\
(7 / 12)\end{array}$ & $\begin{array}{c}86.7 \% \\
(13 / 15)\end{array}$ & $\begin{array}{l}76.5 \% \\
(26 / 34)\end{array}$ \\
\hline
\end{tabular}

Nominees Rated as Well Qualified

Notes: Percentages with observations divided by totals in parentheses. $*$ denotes within-president $\mathrm{Chi}^{2}$ test significant at $\mathrm{p}<.10$. $* *$ denotes within-president $\mathrm{Chi}^{2}$ test significant at $\mathrm{p}<.05$.

seven $(29 \%)$ earned an Ivy League law degree (four of those degrees were from Harvard, two from Yale, and one from Penn). A somewhat higher proportion of the men in Obama's appointment cohort (35\%) earned their J.D. from an Ivy League institution.

Another way that the qualifications of judicial nominees are assessed is through the ratings assigned by the American Bar Association (ABA). Nominees are evaluated on the basis of three factors: temperament, integrity, and competence. The ABA's role in rating has been a longstanding part of the nomination process and has received its share of criticism for lack of transparency and bias against certain kinds of nominees. ${ }^{24}$ Generally, the White House sends the names of nominees to the ABA Standing Committee for evaluation, though under the George W. Bush administration, this was done instead by the Senate Judiciary Committee. ${ }^{25}$ During the Obama administration, White House officials returned to the practice prior to Bush and consulted with the ABA early in the nomination process. News reports at the time alleged that ABA ratings of "not qualified" may have scuttled the nominations of as many as 14 individuals considered by Obama, most of whom were women and/or racial minorities; a much higher proportion of Obama's nominees were rated as "not qualified" than nominees put forward by either Bush or Clinton. ${ }^{26}$

Table 2 shows the breakdown in ratings among Obama's nominees. Among those nominees who were ultimately confirmed, 70.8 percent of women and 87 percent of men nominated by Obama to sit on the federal appellate courts were

female nominees to the lower federal courts were graduates of an elite law school, as compared to their male peers. Slotnick, supra note 3, at 286.

24 See generally, Maya Sen, How Judicial Qualification Ratings May Disadvantage Minority and Female Candidates, 2 J. L. \& Cts. 33(2014); Susan Navarro Smelcer et al., Bias and the Bar: Evaluating the ABA Ratings of Federal Judicial Nominees, 65 Pol. Res. Q. 827 (2012).

25 Amy Steigerwalt, Battle Over the Bench: Senators, Interest Groups, and Lower Court Confirmations, 99-100 (2010).

26 Charlie Savage, Ratings Shrink President's List for Judgeships, N.Y. Times (Nov. 22, 2011), https://www.nytimes.com/2011/11/23/us/politics/screening-panel-rejects-manyobama-picks-for-federal-judgeships.html [https://perma.cc/K5G6-GECX]. 
Table 3: Law Clerk Experience.

\begin{tabular}{|c|c|c|c|c|c|c|c|c|}
\hline & \multicolumn{2}{|c|}{ All Judges } & \multicolumn{2}{|c|}{ Women } & \multicolumn{2}{|c|}{ Racial Minority } & \multicolumn{2}{|c|}{ White } \\
\hline & $\begin{array}{c}\text { All } \\
\text { women }\end{array}$ & $\begin{array}{c}\text { All } \\
\text { men }\end{array}$ & $\begin{array}{c}\text { Nonwhite } \\
\text { women }\end{array}$ & $\begin{array}{c}\text { White } \\
\text { women }\end{array}$ & Women & Men & Women & Men \\
\hline Obama & $\begin{array}{c}66.7 \% \\
(16 / 24) \\
\end{array}$ & $\begin{array}{c}70.9 \% \\
(22 / 31) \\
\end{array}$ & $\begin{array}{c}20 \% * * \\
(1 / 5) \\
\end{array}$ & $\begin{array}{c}78.9 \% * * \\
(15 / 19) \\
\end{array}$ & $\begin{array}{c}20 \% * \\
(1 / 5) \\
\end{array}$ & $\begin{array}{c}64.3 \% * \\
(9 / 14) \\
\end{array}$ & $\begin{array}{r}78.9 \% \\
(15 / 19) \\
\end{array}$ & $\begin{array}{r}76.5 \% \\
(13 / 17) \\
\end{array}$ \\
\hline GWB & $\begin{array}{c}58.8 \% \\
(10 / 17) \\
\end{array}$ & $\begin{array}{c}44.2 \% \\
(19 / 43) \\
\end{array}$ & $\begin{array}{l}33 \% \\
(1 / 3) \\
\end{array}$ & $\begin{array}{l}64.3 \% \\
(9 / 14) \\
\end{array}$ & $\begin{array}{l}33 \% \\
(1 / 3) \\
\end{array}$ & $\begin{array}{l}60 \% \\
(3 / 5) \\
\end{array}$ & $\begin{array}{l}64.3 \% \\
(9 / 14) \\
\end{array}$ & $\begin{array}{r}42.1 \% \\
(16 / 38) \\
\end{array}$ \\
\hline Clinton & $\begin{array}{c}40 \% \\
(8 / 20)\end{array}$ & $\begin{array}{c}47.8 \% \\
(22 / 46)\end{array}$ & $\begin{array}{l}60 \% \\
(3 / 5)\end{array}$ & $\begin{array}{l}33.3 \% \\
(5 / 15)\end{array}$ & $\begin{array}{l}60 \% \\
(3 / 5)\end{array}$ & $\begin{array}{c}25 \% \\
(3 / 12)\end{array}$ & $\begin{array}{c}33 \% \\
(5 / 15)\end{array}$ & $\begin{array}{r}55.9 \% \\
(19 / 34) \\
\end{array}$ \\
\hline
\end{tabular}

Nominees with clerkship experience at any level

Notes: Percentages with observations divided by totals in parentheses. * denotes within-president $\mathrm{Chi}^{2}$ test significant at $\mathrm{p}<.10{ }^{* *}$ denotes within-president $\mathrm{Chi}^{2}$ test significant at $\mathrm{p}<.05$.

rated as "well qualified" by the American Bar Association. Minority women were viewed less favorably in these ratings than their white female counterparts; three of Obama's five nonwhite female nominees were rated as merely "qualified," but nearly 80 percent of Obama's white female nominees were deemed to be "well qualified." Nonwhite women fared more poorly than nonwhite men, as 85 percent of the latter group received "well qualified" ratings (a statistically significant difference at $p$ $<.05)$. This is broadly consistent with other work that finds evidence of systemic disadvantages in the ABA rating scheme for racial minorities and women, though these studies did not examine women of color separately. ${ }^{27}$

Relative to George W. Bush, a comparable percentage of Obama's female nominees were rated by ABA as "well qualified" (both at about 70\%). However, more of Clinton's female nominees had "well qualified" ratings than did Obama's. The trend for nonwhite women was also different under both Bush and Clinton, with more nonwhite women than men receiving "well qualified" ratings.

\section{LAW CLERK EXPERIENCE}

Experience as a law clerk can serve as a valuable stepping-stone to coveted legal jobs like judgeships and is often considered a marker of prestige and quality. Frankel observes that clerkships "can be a career-defining job for the elite law school graduates who win the intense competition for these posts." ${ }^{28}$ Historically, white

27 Susan B. Haire, Rating the Ratings of the American Bar Association Standing Committee on Federal Judiciary, 22 Just. Sys. J. 1, 8 (2001); Sen, supra note 24, at 34; Maya Sen, Minority Judicial Candidates Have Changed: The ABA Ratings Gap Has Not, 98 Judicature 46, 51-53 (2014).

28 Alison Frankel, Breaking the Law Clerks' Code of Silence: The Sexual Misconduct Claims Against Judge Kozinski, Reuters (Dec. 13, 2017, 4:36 PM), https://www. reuters.com/article/us-otc-kozinski/breaking-the-law-clerks-code-of-silence-the-sexualmisconduct-claims-against-judge-kozinski-idUSKBN1E72YX [https://perma.cc/ K2GX-6NT4]. Recent sexual harassment allegations about a federal judge targeting his female law clerks have highlighted the vulnerability of clerks in general and female clerks in particular, which tends to advantage men. Dara E. Purvis, When Judges Prey 
men have been the most common recipients of these opportunities, particularly with respect to Supreme Court clerkships. ${ }^{29}$ In one recent study of federal district court nominees, Sen finds that clerkship experience is much more common among African American district court nominees in the post-Clinton era, though there is not much change in women's experience over time. ${ }^{30}$

Overall, it appears that the Obama administration valued nominees with law clerk experience more than previous presidents. Nearly 70 percent of his appellate nominees had held a clerkship, compared to 48 percent for Bush and 45 percent for Clinton. With respect to women, under Obama, a higher percentage of female nominees $(66.7 \%)$ had previously served as a law clerk compared to women appointed by either Bush (58.8\%) or Clinton (40\%). However, this difference is driven almost entirely by the experiences of Obama's white women nominees, 15 of which had been law clerks. Among women of color, Kara Farnandez Stoll was the only nominee to have law clerk experience, working under Judge Alvin Scholl of the Federal Circuit for one year before moving to private practice. It is notable that, out of the seven black women appointed by the three presidents, only three had law clerk experience, and none of these women was appointed by Obama. In comparison, nearly two-thirds of the minority men (comprising 5 African Americans, 2 Latinos, and 1 Asian-American man) appointed by Obama had law clerk experience.

\section{JUdicial and PROSECUTORIAL EXPERIENCE}

In their review of Obama's judicial legacy, Slotnick, Schiavoni, and Goldman observe that Obama selected the highest proportion of individuals whose previous position before nomination was as a state or federal judge, as well as a record number of former prosecutors. ${ }^{31}$ As seen in Table $4 \mathrm{a}$, half of all women and 54 percent of men in Obama's appellate appointment cohort had prior judicial experience. However, looking among the female nominees, judicial experience was more common among women of color (80\%) than for white women nominees (about $42 \%$ ). Two of the five minority women (Bernice Donald and Jacqueline Nguyen) served in both state and federal judicial roles, but none of the 19 white female nominees had this experience.

When in viewed in comparison with the two prior cohorts of presidential appointees, it appears that, under both Democratic and Republican administrations, prior judicial experience has been something like an unofficial requirement for women of color. ${ }^{32}$ Under Clinton, all 5 nonwhite women had some previous judicial experience, and only two-thirds of white women had served as a judge. While Bush appointed fewer minority women (3), two of those three had prior judicial experience, compared to 57 percent of white women. ${ }^{33}$

on Clerks, N.Y. Times: Opinion (Dec. 12, 2017), https://www.nytimes.com/2017/12/12/ opinion/law-schools-alex-kozinski.html [https://perma.cc/JBT4-THWN].

29 Tony Mauro, Diversity and Supreme Court Law Clerks, 98 Marq. L. Rev. 361, 364-365 (2014).

30 Sen, supra note 27, at 50.

31 Slotnick et al., supra note 5, at 399.

32 Haire \& Moyer, supra note 1, at 63.

33 None of these bivariate relationships are statistically significant, however. 
Table 4a: Judicial Experience.

\begin{tabular}{|c|c|c|c|c|c|c|c|c|}
\hline & \multicolumn{2}{|c|}{ All Judges } & \multicolumn{2}{c|}{ Women } & \multicolumn{2}{c|}{ Racial Minority } & \multicolumn{2}{c|}{ White } \\
\hline \multirow{2}{*}{ All } & $\begin{array}{c}\text { All } \\
\text { women }\end{array}$ & $\begin{array}{c}\text { Nonwhite } \\
\text { women }\end{array}$ & $\begin{array}{c}\text { White } \\
\text { women }\end{array}$ & Women & Men & Women & Men \\
\hline \multirow{2}{*}{ Gbama } & $\begin{array}{c}50 \% \\
(12 / 24)\end{array}$ & $\begin{array}{c}54.8 \% \\
(17 / 31)\end{array}$ & $\begin{array}{c}80 \% \\
(4 / 5)\end{array}$ & $\begin{array}{c}42.1 \% \\
(8 / 19)\end{array}$ & $\begin{array}{c}80 \% \\
(4 / 5)\end{array}$ & $\begin{array}{c}64.3 \% \\
(9 / 14)\end{array}$ & $\begin{array}{c}42.1 \% \\
(8 / 19)\end{array}$ & $\begin{array}{c}47.1 \% \\
(8 / 17)\end{array}$ \\
\hline \multirow{2}{*}{ Clinton } & $58.8 \%$ & $51.2 \%$ & $\begin{array}{c}66.7 \% \\
(2 / 3)\end{array}$ & $\begin{array}{c}57.1 \% \\
(8 / 14)\end{array}$ & $\begin{array}{c}66.7 \% \\
(2 / 3)\end{array}$ & $\begin{array}{c}80 \% \\
(4 / 5)\end{array}$ & $\begin{array}{c}57 \% \\
(8 / 14)\end{array}$ & $\begin{array}{c}47.4 \% \\
(18 / 38)\end{array}$ \\
\hline \multirow{2}{*}{$\begin{array}{c}(10 / 17) \\
(22 / 43)\end{array}$} & $75 \% * *$ & $43.5 \% * *$ & $100 \%$ & $66.7 \%$ & $\begin{array}{c}100 \% \\
(5 / 5)\end{array}$ & $\begin{array}{c}75 \% \\
(9 / 12)\end{array}$ & $\begin{array}{c}66.7 \% \\
(10 / 15)\end{array}$ & $\begin{array}{c}32.4 \% \\
(11 / 34)\end{array}$ \\
\hline
\end{tabular}

Table 4b: Prosecutorial Experience.

\begin{tabular}{|c|c|c|c|c|c|c|c|c|}
\hline & \multicolumn{2}{|c|}{ All Judges } & \multicolumn{2}{|c|}{ Women } & \multicolumn{2}{|c|}{ Racial Minority } & \multicolumn{2}{|c|}{ White } \\
\hline & $\begin{array}{c}\text { All } \\
\text { women }\end{array}$ & $\begin{array}{c}\text { All } \\
\text { men }\end{array}$ & $\begin{array}{c}\text { Nonwhite } \\
\text { women }\end{array}$ & $\begin{array}{c}\text { White } \\
\text { women }\end{array}$ & Women & Men & Women & Men \\
\hline Obama & $\begin{array}{r}41.7 \% \\
(10 / 24) \\
\end{array}$ & $\begin{array}{c}45.2 \% \\
(14 / 31) \\
\end{array}$ & $\begin{array}{l}40 \% \\
(2 / 5) \\
\end{array}$ & $\begin{array}{l}42.1 \% \\
(8 / 19) \\
\end{array}$ & $\begin{array}{l}40 \% \\
(2 / 5) \\
\end{array}$ & $\begin{array}{c}50 \% \\
(7 / 14) \\
\end{array}$ & $\begin{array}{c}42.1 \% \\
(8 / 19) \\
\end{array}$ & $\begin{array}{l}41.2 \% \\
(7 / 17) \\
\end{array}$ \\
\hline GWB & $\begin{array}{c}23.5 \% * \\
(4 / 17) \\
\end{array}$ & $\begin{array}{l}48.8 \% * \\
(21 / 43)\end{array}$ & $\begin{array}{c}33.3 \% \\
(1 / 3) \\
\end{array}$ & $\begin{array}{l}21.4 \% \\
(3 / 14) \\
\end{array}$ & $\begin{array}{c}33.3 \% \\
(1 / 3) \\
\end{array}$ & $\begin{array}{l}40 \% \\
(2 / 5) \\
\end{array}$ & $\begin{array}{c}21.4 \% * \\
(3 / 14) \\
\end{array}$ & $\begin{array}{c}50 \% * \\
(19 / 38) \\
\end{array}$ \\
\hline Clinton & $\begin{array}{c}35 \% \\
(7 / 20)\end{array}$ & $\begin{array}{c}34.8 \% \\
(16 / 46)\end{array}$ & $\begin{array}{c}80 \% * * \\
(4 / 5)\end{array}$ & $\begin{array}{c}20 \% * * \\
(3 / 15)\end{array}$ & $\begin{array}{c}80 \% * \\
(4 / 5)\end{array}$ & $\begin{array}{c}33.3 \% * \\
(4 / 12)\end{array}$ & $\begin{array}{c}20 \% \\
(3 / 15)\end{array}$ & $\begin{array}{r}35.3 \% \\
(12 / 34)\end{array}$ \\
\hline
\end{tabular}

Table 4c: Judicial and Prosecutorial Experience.

\begin{tabular}{|c|c|c|c|c|c|c|c|c|}
\hline & \multicolumn{2}{|c|}{ All Judges } & \multicolumn{2}{c|}{ Women } & \multicolumn{2}{c|}{ Racial Minority } & \multicolumn{2}{c|}{ White } \\
\hline \multirow{5}{*}{ Obama } & $\begin{array}{c}\text { All } \\
\text { women }\end{array}$ & $\begin{array}{c}\text { All } \\
\text { men }\end{array}$ & $\begin{array}{c}\text { Nonwhite } \\
\text { women }\end{array}$ & $\begin{array}{c}\text { White } \\
\text { women }\end{array}$ & Women & Men & Women & Men \\
\hline \multirow{3}{*}{ GWB } & $\begin{array}{c}29.2 \% \\
(7 / 24)\end{array}$ & $\begin{array}{c}25.8 \% \\
(8 / 31)\end{array}$ & $\begin{array}{c}40 \% \\
(2 / 5)\end{array}$ & $\begin{array}{c}26.3 \% \\
(5 / 19)\end{array}$ & $\begin{array}{c}40 \% \\
(2 / 5)\end{array}$ & $\begin{array}{c}35.7 \% \\
(5 / 14)\end{array}$ & $\begin{array}{c}26.3 \% \\
(5 / 19)\end{array}$ & $\begin{array}{c}17.7 \% \\
(3 / 17)\end{array}$ \\
\hline \multirow{2}{*}{ Clinton } & $11.8 \%$ & $23.3 \%$ & $33.3 \%$ & $7.1 \%$ & $33.3 \%$ & $20 \%$ & $7.1 \%$ & $23.7 \%$ \\
$(2 / 17)$ & $(10 / 43)$ & $(1 / 3)$ & $(1 / 14)$ & $(1 / 3)$ & $(1 / 5)$ & $(1 / 14)$ & $(9 / 38)$ \\
\hline & $35 \%$ & $17.4 \%$ & $80 \% * *$ & $20 \% * *$ & $80 \% *$ & $33.3 \% *$ & $20 \%$ & $11.8 \%$ \\
$(7 / 20)$ & $(8 / 46)$ & $(4 / 5)$ & $(3 / 15)$ & $(4 / 5)$ & $(4 / 12)$ & $(3 / 15)$ & $(4 / 34)$ \\
\hline
\end{tabular}

Notes: Percentages with observations divided by totals in parentheses. ${ }^{*}$ denotes within-president $\mathrm{Chi}^{2}$ test significant at $\mathrm{p}<.10$. $* *$ denotes within-president $\mathrm{Chi}^{2}$ test significant at $\mathrm{p}<.05$.

Among those who served as prosecutors prior to being nominated by Obama, Table $4 \mathrm{~b}$ shows little difference across most gender-race cohorts; white women, nonwhite women, and white men all had similar levels of experience $(42 \%, 40 \%$, and $42 \%$, respectively), while half of the nonwhite men nominated by Obama had previously served as a prosecutor at the state or federal level. This emphasis on prosecutorial experience was not viewed favorably by some liberal interest groups. For instance, a 2013 news report highlighted concerns raised by the Alliance for Justice when Obama's appointments of former prosecutors to district and circuit 
Table 5a: Private Practice Experience.

\begin{tabular}{|c|c|c|c|c|c|c|c|c|}
\hline & \multicolumn{2}{|c|}{ All Judges } & \multicolumn{2}{|c|}{ Women } & \multicolumn{2}{|c|}{ Racial Minority } & \multicolumn{2}{|c|}{ White } \\
\hline & $\begin{array}{c}\text { All } \\
\text { women }\end{array}$ & $\begin{array}{l}\text { All } \\
\text { men }\end{array}$ & $\begin{array}{c}\text { Nonwhite } \\
\text { women }\end{array}$ & $\begin{array}{c}\text { White } \\
\text { women }\end{array}$ & Women & Men & Women & Men \\
\hline Obama & $\begin{array}{c}83.3 \% \\
(20 / 24) \\
\end{array}$ & $\begin{array}{c}87.1 \% \\
(27 / 31) \\
\end{array}$ & $\begin{array}{l}80 \% \\
(4 / 5) \\
\end{array}$ & $\begin{array}{c}84.2 \% \\
(16 / 19) \\
\end{array}$ & $\begin{array}{l}80 \% \\
(4 / 5) \\
\end{array}$ & $\begin{array}{c}100 \% \\
(14 / 14)\end{array}$ & $\begin{array}{c}84.2 \% \\
(16 / 19)\end{array}$ & $\begin{array}{r}76.5 \% \\
(13 / 17) \\
\end{array}$ \\
\hline GWB & $\begin{array}{c}82.4 \% \\
(14 / 17) \\
\end{array}$ & $\begin{array}{c}90.7 \% \\
(39 / 43) \\
\end{array}$ & $\begin{array}{c}66.7 \% \\
(2 / 3) \\
\end{array}$ & $\begin{array}{c}85.7 \% \\
(12 / 14) \\
\end{array}$ & $\begin{array}{c}66.7 \% \\
(2 / 3) \\
\end{array}$ & $\begin{array}{l}80 \% \\
(4 / 5) \\
\end{array}$ & $\begin{array}{c}85.7 \% \\
(12 / 14) \\
\end{array}$ & $\begin{array}{r}92.1 \% \\
(35 / 38) \\
\end{array}$ \\
\hline Clinton & $\begin{array}{c}85 \% \\
(17 / 20) \\
\end{array}$ & $\begin{array}{r}89.1 \% \\
(41 / 46) \\
\end{array}$ & $\begin{array}{l}80 \% \\
(4 / 5) \\
\end{array}$ & $\begin{array}{c}86.7 \% \\
(13 / 15) \\
\end{array}$ & $\begin{array}{l}80 \% \\
(4 / 5) \\
\end{array}$ & $\begin{array}{c}91.7 \% \\
(11 / 12) \\
\end{array}$ & $\begin{array}{c}86.7 \% \\
(13 / 15) \\
\end{array}$ & $\begin{array}{r}88.2 \% \\
(30 / 34) \\
\end{array}$ \\
\hline
\end{tabular}

Table 5b: Academic Experience.

\begin{tabular}{|c|c|c|c|c|c|c|c|c|}
\hline & \multicolumn{2}{|c|}{ All Judges } & \multicolumn{2}{c|}{ Women } & \multicolumn{2}{c|}{ Racial Minority } & \multicolumn{2}{c|}{ White } \\
\hline \multirow{2}{*}{ Obama } & $\begin{array}{c}\text { All } \\
\text { women }\end{array}$ & $\begin{array}{c}\text { All } \\
\text { men }\end{array}$ & $\begin{array}{c}\text { Nonwhite } \\
\text { women }\end{array}$ & $\begin{array}{c}\text { White } \\
\text { women }\end{array}$ & Women & Men & Women & Men \\
\hline \multirow{2}{*}{ GWB } & $\begin{array}{c}25 \% \\
(6 / 24)\end{array}$ & $\begin{array}{c}35.5 \% \\
(11 / 31)\end{array}$ & $\begin{array}{c}20 \% \\
(1 / 5)\end{array}$ & $\begin{array}{c}26.3 \% \\
(5 / 19)\end{array}$ & $\begin{array}{c}20 \% \\
(1 / 5)\end{array}$ & $\begin{array}{c}35.7 \% \\
(5 / 14)\end{array}$ & $\begin{array}{c}26.3 \% \\
(5 / 19)\end{array}$ & $\begin{array}{c}35.3 \% \\
(6 / 17)\end{array}$ \\
\hline \multirow{2}{*}{ Clinton } & $29.4 \%$ & $44.2 \%$ & $\begin{array}{c}66.7 \% \\
(5 / 17)\end{array}$ & $\begin{array}{c}21.4 \% \\
(19 / 43)\end{array}$ & $\begin{array}{c}66.7 \% \\
(2 / 3)\end{array}$ & $\begin{array}{c}20 \% \\
(1 / 5)\end{array}$ & $\begin{array}{c}21.4 \% \\
(3 / 14)\end{array}$ & $\begin{array}{c}47.4 \% \\
(18 / 38)\end{array}$ \\
\hline & $\begin{array}{c}35 \% \\
(7 / 20)\end{array}$ & $\begin{array}{c}28.3 \% \\
(13 / 46)\end{array}$ & $\begin{array}{c}20 \% \\
(1 / 5)\end{array}$ & $\begin{array}{c}40 \% \\
(6 / 15)\end{array}$ & $\begin{array}{c}20 \% \\
(1 / 5)\end{array}$ & $\begin{array}{c}33.3 \% \\
(4 / 12)\end{array}$ & $\begin{array}{c}40 \% \\
(6 / 15)\end{array}$ & $\begin{array}{c}26.5 \% \\
(9 / 34)\end{array}$ \\
\hline
\end{tabular}

Notes: Percentages with observations divided by totals in parentheses. ${ }^{*}$ denotes within-president $\mathrm{Chi}^{2}$ test significant at $\mathrm{p}<.10$. $* *$ denotes within-president $\mathrm{Chi}^{2}$ test significant at $\mathrm{p}<.05$.

court judgeships officially surpassed the numbers achieved by George W. Bush or Ronald Reagan. ${ }^{34}$

Of course, some nominees' career paths have taken them through both prosecutorial and judicial roles..$^{35}$ Overall, seven of President Obama's 15 appointees with both judicial and prosecutorial experience were women, a record identical to that of Clinton. In contrast, only two of George W. Bush's 12 nominees with both types of experience were women.

If prior judicial experience is more likely among women of color than white women, is this also the case for experience as both a judge and a prosecutor? The answer appears to be yes. Under Obama, 40 percent of nonwhite women had both judicial and prosecutorial experience, compared to about a quarter of white women. ${ }^{36}$ This trend holds for Bush and Clinton's appointments as well.

\footnotetext{
34 Bob Egelko, Obama Nominations Heavy on Ex-prosecutors, SFGate (Feb. 3, 2013, 11:40 PM), https://www.sfgate.com/nation/article/Obama-nominations-heavy-on-exprosecutors-4248122.php [https://perma.cc/M4SD-USBJ].

35 See supra Table 4c.

36 Of this group of women, one was the first Asian-American woman appointed to an appellate position: Jacqueline Nguyen.
} 


\section{E. PRIVATE PRACTICE}

Outside of previous work as a judge, prosecutor, or law clerk, private practice has been a common training ground for federal judges, in spite of longstanding problems related to diversity and inclusion. ${ }^{37}$ Indeed, the vast majority of Obama female nominees $(83 \%)$ had prior experience in private practice, similar to women appointed by the two previous presidents $(82 \%$ for Bush nominees and $85 \%$ for Clinton nominees). Private practice experience was shared by four out of the five nonwhite women Obama appointed, as well as for 16 out of the 19 white women he seated. Interestingly, the white men appointed by Obama were less likely to have private practice experience than any other race-gender cohort, though the difference is not statistically significant.

\section{F. ACADEMIA}

Less common than private practice experience is previous experience in academia. Slotnick, Schiavoni, and Goldman show that after Reagan, presidents have tended to nominate proportionately fewer law professors to appeals court positions, perhaps as a reaction to the defeat of Robert Bork (a former Yale Law professor) in his nomination to the Supreme Court. ${ }^{38}$

Table $5 \mathrm{~b}$ displays the breakdown of nominees with academic experience across Obama, Bush, and Clinton. In terms of absolute numbers, Obama was somewhat less apt to select nominees who had previously worked in academia, relative to his predecessors. (Twenty of Clinton's appointees and 24 of Bush's appointees had prior experience in academia, compared to 17 of Obama's appointees.)

Of Obama's nominees with academic experience, four of the six women who worked in law schools did so as adjunct, short-term, or visiting faculty. Bernice Donald, the only nonwhite woman appointed by Obama to have had academic experience, served as an adjunct professor for three years at Memphis State Law School (now University of Memphis Humphreys School of Law). The only women who served in tenure track positions were both white and taught at elite institutions: Pamela Harris taught at the University of Pennsylvania Law, and Cornelia Pillard was on the faculty at Georgetown Law. In contrast, more than half of Obama's male nominees with academic experience had full-time, tenure track positions at law schools. The difference in type of academic appointment may reflect broader trends in higher education, whereby increases in racial and gender diversity have tended to come in non-tenure track positions ${ }^{39}$ and where structures and norms still tend to favor white men. ${ }^{40}$ These differences in academic experience could also reflect

37 Haire \& Moyer, supra note 1, at 40; Am. Bar Ass'n: Comm'n on Women in the Profession, A Current Glance at Women in the Law 2016 at 2 (2016), https://www.americanbar. org/content/dam/aba/administrative/women/current_glance_statistics_may2016.pdf [https://perma.cc/3YZA-CDP7]; Epner, supra note 9, at xi.

38 Slotnick et al., supra note 5, at 400.

39 Colleen Flaherty, More Faculty Diversity, Not on Tenure Track, Inside Higher Ed (Aug. 22, 2016), https://www.insidehighered.com/news/2016/08/22/study-finds-gains-facultydiversity-not-tenure-track [https://perma.cc/W2L5-WQJF].

40 See, e.g., Maritza I. Reyes et al., Reflections on Presumed Incompetent: The Intersections of Race and Class for Women in Academia Symposium - The Plenary Panel, 29 Berkeley 
a gendered nomination strategy by the Obama administration, in which women with a track record of academic publications might be seen as offering too much of a "paper trail" for the confirmation process. There is no question that, over the past three presidential administrations, the Senate's confirmation process for lower court nominees has changed in ways that have made it more contentious; the next section addresses what these developments have meant for women nominated to be appellate judges.

\section{Running the Gauntlet: The Senate Confirmation PROCESS}

\section{A. Changes in the Senate Confirmation Process}

Obama's strategy to diversify the bench coincided with a time of intense polarization ${ }^{41}$ and partisan conflict resulting in unprecedented levels of obstruction and delay in the Senate confirmation process. ${ }^{42}$ To understand how the experiences of Obama's female nominees compared with others in their appointment cohort, it is vital to note several key developments that affected prospects for all nominees.

First, appeals court nominees have increasingly faced obstruction and delay in their confirmations. To operationalize these concepts, Slotnick and colleagues created a measure that is the number of nominations unconfirmed plus the number of nominations that took more than 180 days from nomination to confirmation. The index runs from 0 (no obstruction and/or delay) to 1 (complete obstruction and/or delay). Figure 2 shows how, from 1978 to 2016, the index of obstruction and delay goes from its lowest level in the late 1970s to complete obstruction and delay by the $114^{\text {th }}$ Congress when the Republicans won control of the Senate. ${ }^{43}$ As Senator McConnell has himself acknowledged, ${ }^{44}$ the new Republican majority adopted a strategy that would prevent Obama from naming any judges during the last two years. (This was in addition to McConnell's more public decision to prevent

J. Gender L. \& Just. 195, (2014).

$41 \quad$ See e.g., Christopher Hare \& Keith T. Poole, The Polarization of Contemporary American Politics, 46 Polity 411 (2014); Andrew Kohut et al., Pew Res. Ctr., Trends in American Values: 1987-2012-Partisan Polarization Surges in Bush, Obama Years (2012), https:// assets.pewresearch.org/wp-content/uploads/sites/5/legacy-pdf/06-04-12\%20Values\%20 Release.pdf [https://perma.cc/8JU7-PJ8L].

42 Slotnick et al., supra note 5, at 375-380. The obstruction and delay in confirmation was not limited to judicial nominees. Slotnick, Schiavoni, and Goldman point to Republican filibusters of nominees to the EPA, National Labor Relations Board, Department of Labor, and the Bureau of Alcohol, Tobacco, Firearms, and Explosives, Department of Defense, Office of Personnel Management, and the Federal Housing Finance Agency. Elliot E. Slotnick et al., Writing the Book of Judges: Part 2: Confirmation Politics in the 113th Congress, 4 J. L. \& Cts. 187, 191 (2016).

43 The measure does not take into account nominations to the Federal Circuit, so it does not include the confirmation of Kara Farnandez Stoll in January 2015.

44 Ed Mazza, Mitch McConnell Brags About Blocking Obama for 2 Years, Then Laughs About It, HuffPost (Dec. 13, 2019, 3:28 AM), https://www.huffpost.com/entry/mitchmcconnell-blocks-obama-laughs_n_5df32430e4b0deb78b517322 [https://perma.cc/ WPE5-RK8Q]. 


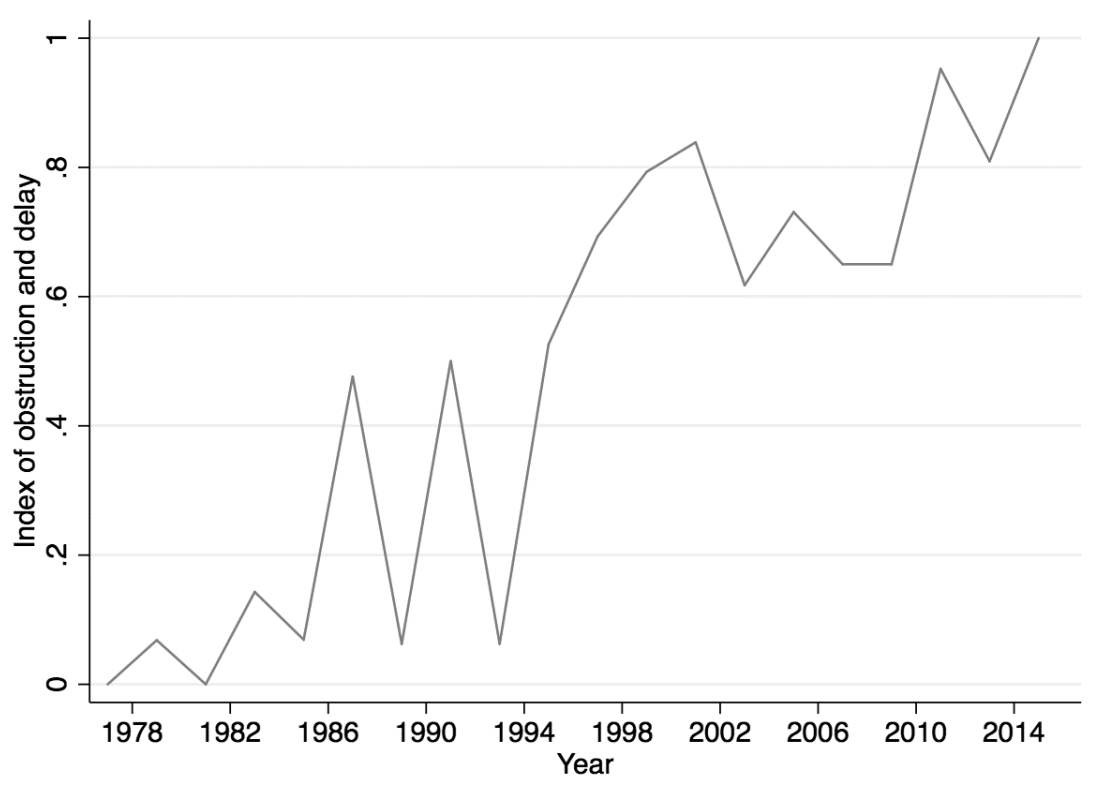

Figure 2: Index of Obstruction and Delay, 1978-2016 45

Supreme Court nominee Merrick Garland from receiving a vote in the Senate.) One Democratic staffer explained:

I think that what Mitch McConnell understands is that judicial nominations is a niche area that has a lot of influence over policy but he will never lose any votes over it. That's why he did what he did the last two years in filling judicial vacancies. That's why he had no compunction against holding up the Obama nominations process in the early years. He's internalized this because he's seen the way that this can advance his political agenda, and he knows that he's not going to get any blowback in the political sphere. ${ }^{46}$

Second, Obama judicial nominees' experiences were impacted by the erosion of norms that had generally worked to ensure the efficient and timely confirmation of lower federal court nominees. Presidents have historically conferred with home state senators of the president's party on the selection of nominees for vacancies within those senators' states; the Senate Judiciary Committee has also utilized the blue slip norm to secure the support of home state senators from both parties. ${ }^{47}$ While there has been some variation in how much deference was afforded to home

\footnotetext{
Slotnick et al., supra note 5, at 379.

46 Slotnick et al., supra note 5, at 383.

47 Elliot E. Slotnick, Appellate Judicial Selection During the Bush Administration: Business as Usual or a Nuclear Winter?, 48 Ariz. L. Rev. 225, 227 (2006).
} 
state senators ${ }^{48}$ scholars have noted an acceleration in the use of retaliatory blue slips through the end of the George W. Bush era and continued under Obama. ${ }^{49}$ Steigerwalt notes a number of examples of senators using the blue slip as a way to seek retribution for past nominees' treatment and concludes that "[s]enatorial courtesy is thus being used as a weapon in institutional (and partisan) fights that look more and more like the normal politicking that characterizes the normal legislative process. $"{ }^{50}$

Another institutional practice that has affected the confirmation process for appeals court nominees is the filibuster. This tool aids the minority party by stopping all Senate business until 60 senators agree to invoke cloture, and it serves as a highly public and dramatic expression of opposition. ${ }^{51}$ As Slotnick, Goldman, and Schiavoni detail, when Obama took office in 2009, Republicans were still smarting from filibuster defeats of two nominees to the DC Circuit (Miguel Estrada and Peter Keisler), and they responded in kind to Obama's efforts to fill the DC vacancies. $^{52}$ Democrats, frustrated by the blockage of Obama's nominees to the DC Circuit and to the National Labor Relations Board, responded to calls from within their own caucus to push for a rules change to end the filibuster. Some of the senators who supported this change were new arrivals to the Senate and had not been in the minority before, while other "institutionalists," like Patrick Leahy, were reluctantly convinced of the necessity of this rule change by the treatment of the DC Circuit nominees. One staffer on the Judiciary Committee pointed in particular to the nomination of a woman named Patricia Millett as a tipping point in changing his mind in favor of filibuster reform:

The filibuster of her nomination really troubled [Sen. Leahy], because he thinks long term .... But the change in demeanor from the summer, where we almost had a rules change ... then we heard positive things being said about Patti Millett ... nothing but positive things ... . "She looks like an excellent candidate" becomes, in November, the same member [John McCain] who'd been involved in the Gang of Fourteen in 2005 came down to the floor [of the Senate] to say 'I deem this an extraordinary circumstance.' Her nomination, a completely noncontroversial nomination. We were trying to work with them [the Republicans]." ${ }_{53}$

48 Sarah A. Binder, Where Do Institutions Come From? Exploring the Origins of the Senate Blue Slip, 21 Stud. Am. Pol. Dev. 1, 6-12 (2007).

49 Steigerwalt, supra note 25, at 63, 193; Slotnick et al., supra note 5, at 369-375.

50 Steigerwalt, supra note 25, at 65-66. Another practice that has increasingly affected increasing levels of confirmation delay is senators' use of holds as leverage for other policy disputes. $I d$. at $84-87$.

51 Id. at 179.

52 Slotnick et al., supra note 42, at 195. A bipartisan group of senators known as the "Gang of 14" secured the confirmation of four Bush appointees to the DC Circuit, including Brett Kavanaugh. Elliot E. Slotnick et al., W. Bush's Judicial Legacy: Mission Accomplished, 92 Judicature 258, 271-272(2009). The coalition formed as a way to prevent Republicans from using the "nuclear option" themselves and preserved the ability of the Democrats to filibusterbut only in "extraordinary circumstances." Slotnick et al., supra note 42, at 190. During the Obama years, however, no similar "gang" emerged to save the filibuster and seat judges.

53 Slotnick et al., supra note 42, at 193. Millet was ultimately confirmed to the DC Circuit after the rules change took effect. 
This episode, and the Democrats' reaction to it, highlight another trend that affected the fates of judicial nominees: eroding trust between the two political parties. ${ }^{54}$

Lastly, Obama's nominees went through the confirmation process in an era in which most floor votes on their nominations would be recorded via roll call. Whereas voice votes tend to indicate action by unanimous consent, ${ }^{55}$ roll call votes put individual members on the record. ${ }^{56}$ Scholars of Congress have observed the declining prevalence of the voice vote in both chambers, finding that electoral pressures influence decisions by members to record their votes. ${ }^{57}$

This shift toward more roll call votes has increasingly affected judicial nominations as well. Since 1965, Supreme Court nominee votes have been recorded, ${ }^{58}$ and studies have identified the 1990s as a turning point for how votes on lower federal court nominations are handled. ${ }^{59}$ Cohen notes that the $105^{\text {th }}$ Congress (under Clinton) represented a highwater mark in roll call votes:

$[\mathrm{N}]$ ominees to federal judgeships were more likely than at any other point in history to be subject to a roll call vote for confirmation .... This may be the result of maneuvering by conservative Republicans; the Republican conference decided early in 1997 that judicial nominees were too important to confirm by voice vote. ${ }^{60}$

Since Clinton, the trend of recording votes on future judges has only accelerated. While only a quarter of Clinton's appellate nominees received a roll call vote, this shot up to 80 percent under Bush and then a staggering 87 percent under Obama. ${ }^{61}$

54 Boyd, Lynch, and Madonna find that after the rules change (although before Republicans won the Senate in the $114^{\text {th }}$ Congress), Obama's judicial nominees were confirmed more quickly than they had before invoking the "nuclear option." Christian L. Boyd et al., Nuclear Fallout: Investigating the Effect of Senate Procedural Reform on Judicial Nominations, 13 Forum 623 (2015).

55 Charles M. Cameron et al., Senate Voting on Supreme Court Nominees: A Neoinstitutional Model, 84 Am. Pol. Sci. Rev. 525 (1990); Lee Epstein et al., The Changing Dynamics of Senate Voting on Supreme Court Nominees, 68 J. Pol. 296 (2006).

56 Oleszek notes that "recorded votes in the Senate usually can be obtained easily; only a sufficient second - one-fifth of the senators present-is needed, with a minimum of 11 required by the Constitution." Walter Oleszek, Congressional Procedures and the Policy Process 236 (6th ed. 2004). He goes on to quote Sen. Robert Byrd, who observed "If any Senator wants a roll call vote around here, he will ultimately get it." Id. at 236.

57 Michael S. Lynch \& Anthony J. Madonna, Viva Voce: Implications from the Disappearing Voice Vote, 1865-1996, 94 Soc. Sci. Q. 530 (2013).

58 Charles M. Cameron et al., Voting for Justices: Change and Continuity in Confirmation Voting 1937-2010, 75 J. Pol. 283, 286 (2013).

59 Scherer et al., supra note 13, at 1027.

60 Lauren M. Cohen, Missing in Action: Interest Groups and Federal Judicial Appointments, 82 Judicature 119, 121 (1998).

${ }_{61}$ The shift toward increased roll call votes coincides with an increase in interest group participation for lower court nominations and increased polarization among senators. Id. at 121-122; Lauren Cohen Bell, Senatorial Discourtesy: The Senate's Use of Delay to Shape the Federal Judiciary, 55 Pol. Res. Q. 589, 591-596 (2002); Cameron et al., supra note 58 , at 285 . 

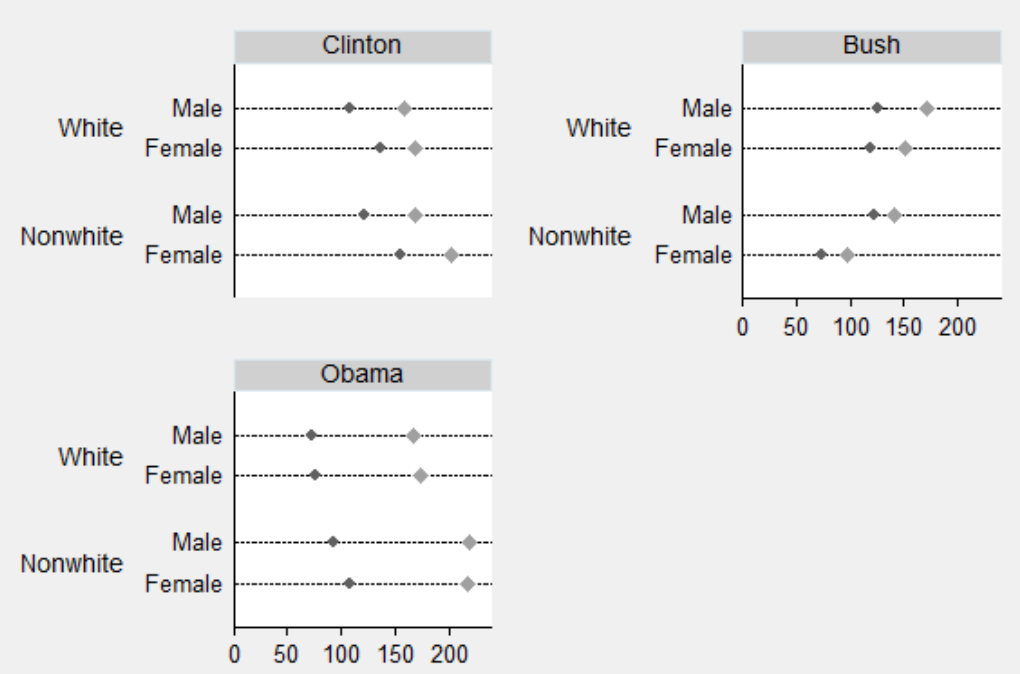

- Days until reported out of committee $\quad$ Days until confirmed by Senate

Figure 3: Committee and Confirmation Delay for Appeals Court Nominees, by Race and Gender.

\section{B. AsSESSING the TREATMENT OF Obama's FeMaLE NomineES IN tHe SENATE}

Taking all of these factors into account, should we expect female nominees under Obama to be treated differently on the basis of their gender and/or race? The existing literature is mixed about the extent to which women and nonwhite nominees experience a lengthier and less successful confirmation process, with some finding a disadvantage for women and racial minorities ${ }^{62}$ and others failing to find any race or gender differences. ${ }^{63}$ To assess this descriptively, I examine a series of outcomes for appeals court nominees who were ultimately confirmed: time from nomination to confirmation, delay at the committee stage, whether a roll call vote was taken, and (for those who received roll call votes) the number of "yes" votes for the nominee.

Figure 3 plots two indicators of delay, the mean number of days a nomination stayed in the Judiciary Committee before being reported out (committee delay) and the mean number of days from nomination to confirmation (confirmation delay) ${ }^{64}$ Looking first at Obama nominees, white men had the least amount of committee and confirmation delay of any race-gender cohort (72 days and 166 days, respectively).

62 E.g., Holmes et al., supra note 14, at 43.

63 E.g., Nicole Asmussen, Female and Minority Judicial Nominees: President's Delight and Senators' Dismay?, 36 Legis. Stud. Q. 591, 609-610 (2011); Wendy L. Martinek et al., To Advise and Consent: The Senate and Lower Federal Court Nominations, 19771998, 64 J. Pol. 337, 358 (2002).

64 The data for committee delay for Bush and Clinton appointees are from Holmes, Shomade, and Hartley. Holmes et al., supra note 14. 


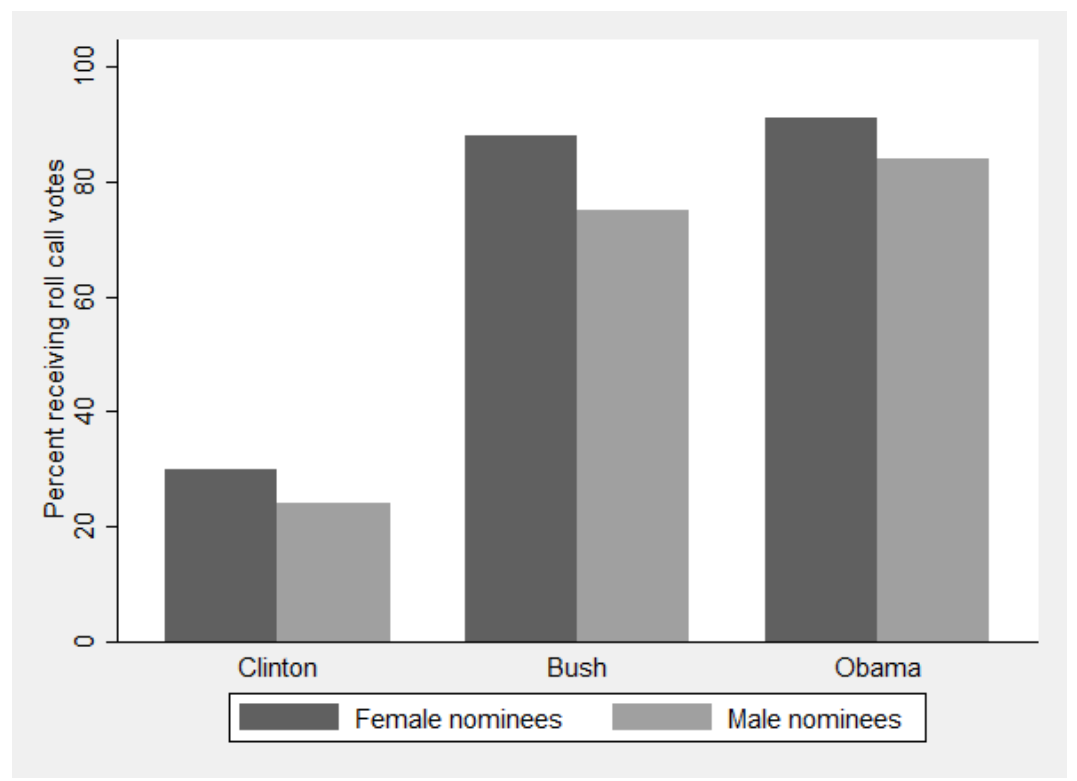

Figure 4: Roll Call Votes for Women Nominees to the U.S. Courts of Appeals.

Note: Within-president differences are not statistically significant at $\mathrm{p}<.05$ for any president.

White women experienced slightly more of both types of delay than white men, waiting about a week longer to be confirmed. However, women of color saw their nominations sit longer in committee than nonwhite men (108 days versus 93 days) and stretch out longer until confirmation than white women (217 days versus 173 days). While none of these differences are statistically significant under a t-test, they are suggestive of a distinctive and potentially more contentious process for minority women, at least those nominated by Democratic presidents. ${ }^{65}$

Overall, Obama's female nominees faced lengthier confirmation times than women nominated by the two previous presidents, waiting an average of 182 days compared to 141 days for Bush female nominees and 176 days for Clinton female nominees. With respect to delay in committee, Obama's female nominees actually fared better than women nominated by either Clinton or Bush. Nominations by Obama took an average of 82 days to be reported out of committee, while it took 110 days for Bush's female nominees and a whopping 141 days for Clinton's female nominees. This largely reflects the different political circumstances for each president related to divided government; Obama had a Democratic-led Judiciary Committee for all but two years of his time in office. ${ }^{66}$

65 Under Obama, the average confirmation delay for black women $(\mathrm{n}=2)$ was 203 days and 226 for Hispanic women $(\mathrm{n}=2)$.

66 However, during 2016, two women and one man were reported favorably out of the Senate Judiciary Committee but not did not receive a vote on the Senate floor. One of the women, Lucy Koh had previously been unanimously confirmed to a federal district court position in 2010 and was the first Korean-American woman confirmed to an Article III judgeship. Slotnick et al., supra note 5, at 399. 

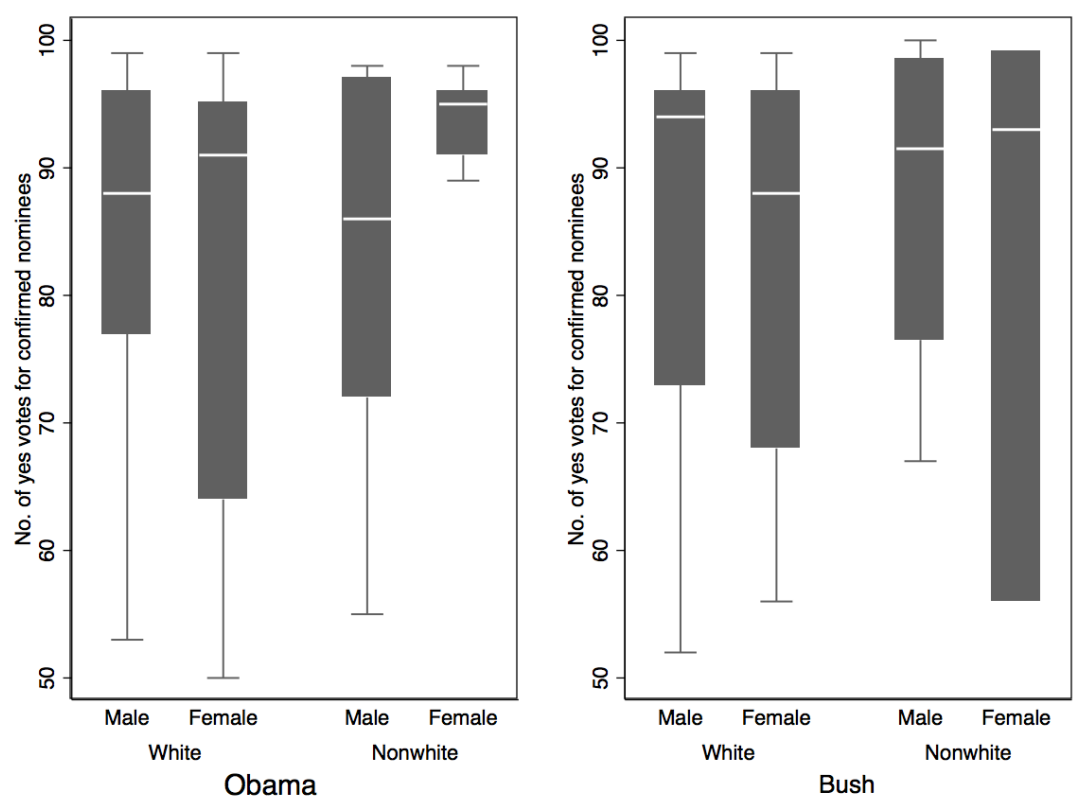

Figure 5: Votes to Confirm Appeals Court Nominees.

Box plots of roll call votes for successful nominees

Note: There were 29 white men, 12 white women, 4 nonwhite men, and 3 nonwhite women appointed by George W. Bush who received a roll call vote. Among successful Obama nominees who received a roll call vote, 15 were white men, 17 white women, 11 nonwhite men, and 5 were nonwhite women.

Moving next to the method of voting on nominees, the literature suggests that both interest groups and the parties would have strong interests in requiring senators to take a recorded vote on Obama's judicial nominees. As noted above, 87 percent of Obama's nominees to the federal appeals courts received a roll call vote (compared to $25 \%$ of Clinton's nominees). How did this vary based on nominee gender? Figure 4 shows the percent of women and men whose nominations received a recorded vote. Apart from the dramatic shift from Clinton to the two more recent presidents, what is notable is that, regardless of party or president, a higher percentage of women are put up for roll call votes than men. Among Obama's successful nominees to the U.S. Courts of Appeals, all five women of color were confirmed via a roll call vote, the highest among any gender-race cohort. (This was also true for all of George W. Bush's nonwhite women.) Seventeen out of 19 white women (89\%), 11 out of 13 nonwhite men (78\%), and 15 out of 17 white men (88\%) nominated by Obama also had roll call votes. Taken together, this suggests that there are perceived electoral benefits related to voting for women nominees and especially so for women of color, consistent with conclusions of past work. ${ }^{67}$ 
Among all those who did receive a recorded vote and who were ultimately confirmed, what differences were there in the number of "yes" votes received? Figure 5 shows a box plot of the distribution of votes to confirm each race-gender cohort of nominees; because of how few Clinton nominees received a roll call vote, the graph compares only Obama and Bush judges. There are some striking differences across groups. Notably, we see very little variation in the number of "yes" votes for minority women appointed by Obama, especially when compared to white women in the same appointment cohort. (The reverse is true for Bush women appointees.) Second, women of color appointed by Obama had the highest median number of "yes" votes of that appointment cohort, which is interesting given their lower ABA ratings. ${ }^{68}$ And while these findings should not be interpreted as representative of all those who were nominated, they do suggest that electoral incentives during the Obama years drove senators of both parties to support minority women's confirmation. It also suggests that the White House may have only opted to select minority women in whom they had great confidence of gaining bipartisan support. More research, using both successful and unsuccessful nominations, is needed to understand these dynamics more fully.

\section{CONCLUSIONS}

In spite of a highly contentious political environment, President Obama was able to make substantial progress toward his stated goal of a federal judiciary that "looks like America." His appointments to the federal appellate bench improved both gender and racial diversity among judges and exceeded the accomplishments of previous presidents who had also declared diversity a goal. ${ }^{69}$ In addition, Obama sought out diversity in professional experiences, yielding more female nominees who had worked as law clerks and prosecutors than previous presidents.

Although this Article is descriptive in its approach, its findings point to a number of directions for future research. First, there is clearly value in adopting an intersectional framework in analyzing the professional backgrounds and confirmation experiences of judicial nominees, because the experiences of women of color are not identical to those of white women or men of color. For instance, among Obama's nonwhite nominees, men were more likely to have held clerkships than women and also were more likely to have served as a prosecutor. And across presidents of both parties, we see that women of color appear to be held to a higher standard than white women in terms of an expectation of prior judicial service. Given that minority women are the most underrepresented race-gender group in both state and federal judiciaries,$^{70}$ this appointment trend is striking.

Future research should explore the underlying causal mechanisms at work for these observed differences. The literature suggests several possibilities. In their

68 Interestingly, the only woman of color nominated by Clinton who received a roll call vote was Sonia Sotomayor, who garnered only 68 "yes" votes in spite of her "well qualified" rating and prior experience as a U.S. District Court judge and prosecutor.

69 Haire \& Moyer, supra note 1, at 131-135.

70 Id. at 77-78; Tracey E. George \& Albert H. Yoon, Am. Constitution Soc'y for Law \& Policy, The Gavel Gap: Who Sits in Judgment on State Courts? at 7 (2016), https:// gavelgap.org/pdf/gavel-gap-report.pdf [https://perma.cc/7NFJ-YDTT]. 
study of appointments to the European Court of Justice, Gill and Jensen find that women are expected to demonstrate particular traits through previous professional experiences, while men are assumed to possess such traits even without comparable types of professional experience. ${ }^{71}$ Alternatively, presidents may opt to select women of color with proven track records as judges as a way to anticipate and head off potential opposition in the Senate. Asmussen's findings suggest that this could be part of a strategy to put pressure on and potentially disadvantage senators of the opposing party. For other kinds of professional experiences, such as law clerkships or academic positions, differences may be a function of structural biases against women and people of color, which then manifest themselves as different career paths for different groups of individuals.

Consistent with press accounts that described President Obama's frustrations about low ABA ratings of his nontraditional nominees, ${ }^{72}$ I find that fewer of Obama's women nominees were rated as "well qualified" than their male counterparts. This difference is largely driven by the ratings of nonwhite women, which are significantly lower than those of white women and nonwhite men. It is likely that the mix of individuals observed in these data - which consist only of confirmed judges - reflects the Obama White House's reaction to how the ABA was assessing other potential nominees. Given the contentious Senate environment, the White House may have opted for nominees who appeared to be less risky, though this may have been hampered by systematic bias in the ABA evaluation process. ${ }^{73}$ More work is needed to understand how the ABA process influences the calculus of presidents in selecting nontraditional nominees.

Additionally, future research should investigate the implications of the shift to more roll call votes for appellate and district court nominations. I find that, over three presidents, women have been more likely than men to receive a roll call vote, and that under Obama, all of his confirmed nonwhite women were subject to a recorded vote on their confirmation. This suggests that, at least among those who were ultimately confirmed, women have been less likely to be viewed as a consensus choice deserving of a voice vote. On the other hand, if the goal is to make the Senate more representative, there may be advantages to putting more confirmation votes on the record.

Going forward, it is evident that the Republican strategy of blocking Obama nominees in the last two years has paid off in terms of vacancies available to be filled by President Trump. Solberg and Diascro note that by the end of Trump's first year in office, he had the opportunity to fill more than double the number of lower court vacancies that Obama inherited when he took office. ${ }^{74}$ Under Senate Majority Leader McConnell, there was a single-minded focus on confirming President Trump's judicial nominees to the Supreme Court and to the lower federal courts, which yielded impressive results for the single-term president. In four years, Trump succeeded in confirming 54 judges to the U.S. Courts of Appeals, more than twothirds of which were white men. Among the 11 women confirmed to the appellate

71 Rebecca D. Gill \& Christian Jensen, Where are the Women? Legal Traditions and Descriptive Representation on the European Court of Justice, 2018 Pol. Grps. \& Idents. 1.

72 Savage, supra note 26.

73 Sen, supra note 24, at 44.

74 Solberg \& Diascro, supra note 5, at 12. 
bench, only two were women of color. ${ }^{75}$ In contrast, nine months into his first year in office, President Biden's circuit court appointees evidenced a dramatic change from his predecessor; not only were all appointees women, but four of the five are women of color. While it remains to be seen how much President Biden will continue to prioritize diversity in his judicial appointments, these judges will join courts reshaped by both the Obama and Trump cohorts and together will influence the development of the law for years to come.

75 Under President Trump, a Latina, Barbara Lagoa, was confirmed to the 11th Circuit, and an Asian woman, Neomi Rao, filled now-Justice Brett Kavanaugh's former seat on the DC Circuit. There were also six nonwhite men, all of which were of Asian descent. 